\title{
Design formulae for a concave convex arc line gear mechanism
}

\author{
Yangzhi Chen and Li Yao \\ School of Mechanical and Automotive Engineering, South China University of Technology, \\ Guangzhou, 510640, People's Republic of China \\ Correspondence to: Yangzhi Chen (meyzchen@scut.edu.cn)
}

Received: 11 July 2016 - Revised: 17 October 2016 - Accepted: 30 October 2016 - Published: 11 November 2016

\begin{abstract}
Line gear is a newly developed gear mechanism with point contact meshing according to space curve meshing theory. This paper proposes a new form of line gear with a couple of concave convex arc tooth profiles. It has four characteristics. First, contact curve of the driving line gear is a cylindrical spiral curve. Second, two axes of a pair of line gears are located in the same plane with an arbitrary angle. Third, at the mesh point, normal tooth profiles of a line gear pair are a couple of inscribed circles. Namely, they form a couple of concave convex tooth profiles. Fourth, the tooth profile of a driving line gear is a convex, that of a driven line gear is a concave, and they are interchangeable. If only consider that the arcs of teeth at meshing point are tangent, the actual tooth surfaces may interfere outside of the meshing point. In this paper, the geometric condition of the tooth surface for a concave convex arc line gear mechanism is derived, and the optimal formulae of the tooth profile parameters are derived on basis of interference proof conditions. Finally, the 3-D modeling and kinematic simulation of line gear pairs show that the proposed line gear pairs can perform transmission normally. The proposed method will extend the application of line gear in the conventional power drive.
\end{abstract}

\section{Introduction}

The concave convex arc line gear (Chen and Yao, 2015) is the line gear with arc tooth profile. The arc gear has advantages in large loading capacity. For instance, Sun et al. (2016) investigated the contact strength of the circular-arc-toothtrace cylindrical gear (C-gear). The results reveal that the $\mathrm{C}$-gear is superior to spur gear and helical gear in the contact strength. The curvilinear tooth gear proposed by Zhang et al. (2016) is superior to spur gear in contact and bending stress. The Double Circular-Arc Helical Gear developed by Wang (2012) can be used for heavily-loaded planetary gear reducer. The circular-arc gear designed by Zhou et al. (2016) can be used for a high-pressure and high-speed gear pump in the aerospace application. Because of the advantages of arc tooth, scholars have used the arc tooth in many different types of gear transmission. They are hyperboloidal-type normal circular-arc gears (Chen et al., 2016), helical gear with triple circular-arc teeth (Xie and Yang, 2014), quadruple-arc Profile Bevel Gears (Ren, 2014), a new kind of gear transmission with circular arc tooth profiles (Chen et al., 2014), etc. All of these belong to cylindrical gear. Arc tooth are also used in bevel gears (Dong and Wang, 2014; Zhang et al., 2011, 2012) and worm (Zhao and Zhang, 2011).

The concave convex arc line gear is a new form of line gear. The early line gear is also called the space curve meshing wheel. It contains a driving wheel, a driven wheel, driving tines and driven tines (Chen et al., 2013b). A cantilever structure is formed by the combination of the tines and the wheel body. Compared with the previous proposed line gear, the concave convex arc line gear has the advantages of arc gear. It is better than the early line gears in loading capacity, bears much lower contact and bending stress. It can be applied to conventional power transmission with larger power. It can be processed by the metal cutting machine tool. Compared with other arc gear, it has the advantages of line gear. It has a large transmission ratio and compact structure. The gear shafts of the gear pair can be crossed at any angle. It means that the conventional gear pair must be a driven wheel corresponding to a driving wheel. The concave convex arc line gear pair can be designed as a driving gear and a corresponding plurality 


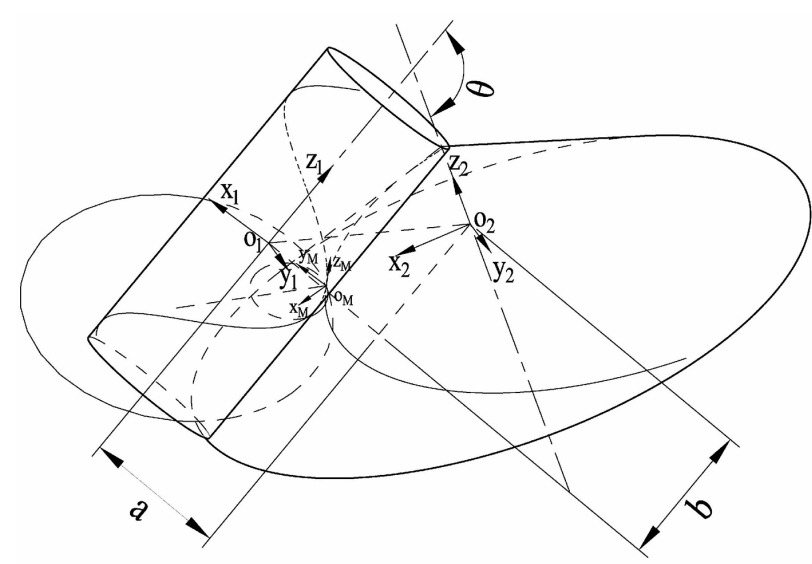

(a)

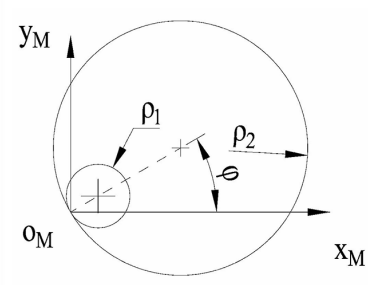

(b)

Figure 1. A pair of concave convex arc line gears at the meshing point.

of driven gears. The minimum tooth number of line gear can reach 1 (Chen, 2014). But it can bear lower contact stress than other conventional forms of arc gear above due to point contact meshing.

The main objective of the work is to improve the original form of line teeth profile, to be capable of applying in conventional power transmission. If the line gear is only the combination of the characteristics of the space curve meshing wheel and the characteristics of the arc gear, it cannot be processed by the metal cutting machine tool. It also shows the importance of the gear profile and the parameter quantization. Moreover, the tooth profile parameters and gear parameters are quantified to provide the basis for the analysis of gear strength. A new design method of the concave convex arc line gear is presented in this paper, aiming to solve the problem of tooth surface geometry interference and the optimization of the tooth profile parameters. In order to verify the feasibility of the parametric design and tooth profile parameters optimization of concave convex arc line gears, this paper presents the specific example and the 3-D modeling and kinematic simulation of the line gear.

\section{Basic design}

A pair of concave convex arc line gears is comprised a driving line gear and a driven line gear. As shown in Fig. 1a, the axes of the driving line gear and the driven line gear are arbitrary angle intersected axes in one plane. The angle is $\theta$. The contact curve of the driving line gear is a spatial cylinder spiral curve, which forms a pair of conjugated space curves with the contact curve of the driven line gear. The contact curves of the driving line gear and the driven line gear are located in $o_{1}-x_{1} y_{1} z_{1}$ and $o_{2}-x_{2} y_{2} z_{2}$ respectively. The distance between $o_{1}$ and $o_{2}$ is the center distance of the driving line gear and the driven line gear. As shown in Fig. 1b, the tooth profiles of line gears are a pair of inscribed circles at the mesh point $o_{\mathrm{M}}$. And the point $o_{\mathrm{M}}$ is also the tangent point. The point $o_{\mathrm{M}}$ and the center points of the circles are located on one straight line, which has an angle of $\phi$ with $x_{\mathrm{M}}$. And $-\boldsymbol{x}_{\mathrm{M}}$ is the secondary normal vector of contact curve of the driving line gear at the mesh point $o_{\mathrm{M}}$ (Chen and Yao, 2015).

The contact curve of the driving line gear, namely a driving contact curve, is set as a spatial cylinder spiral curve. And the parametric equation in $o_{1}-x_{1} y_{1} z_{1}$ is as Eq. (1) (Ding et al., 2012).

$$
\left\{\begin{array}{l}
x_{\mathrm{M}}^{(1)}=m \cos t \\
y_{\mathrm{M}}^{(1)}=m \sin t \\
z_{\mathrm{M}}^{(1)}=n \pi+n t
\end{array}\right.
$$

where $m$ is the helix radius of the cylindrical spiral curve; $n$ is the pitch parameter of the cylindrical spiral curve, denoting the pitch as $p, n=\frac{p}{2 \pi} ; t$ is a parameter variable, $t \in\left[t_{\mathrm{s}}\right.$, $\left.t_{\mathrm{e}}\right]$. The value range of $t$ determines the length of the contact curve. $t_{\mathrm{s}}$ and $t_{\mathrm{e}}$ are the starting and ending values for the meshing point, respectively. $t_{\mathrm{s}}=-\pi-\frac{\Delta t}{2}, t_{\mathrm{e}}=-\pi+\frac{\Delta t}{2}$, $\Delta t=t_{\mathrm{e}}-t_{\mathrm{s}}$. The value of $\Delta t$ satisfies the condition of contact ratio (Chen et al., 2013a): $\varepsilon=\frac{\Delta t N_{1}}{2 \pi} \geq 1 . N_{1}$ and $N_{2}$ are the tooth numbers of the driving line gear and the driven line gear respectively. The value of $\varepsilon$ needs to be preferred. And the value of $\Delta t$ can be deduced. Then the value of $t_{\mathrm{s}}$ and $t_{\mathrm{e}}$ can be determined. Therefore, $\varepsilon, N_{1}, m$ and $n$ are the key factors of the contact curve of the driving line gear.

The contact curve of the driven line gear is conjugated with the driving contact curve, and the equation in $o_{2}-x_{2} y_{2} z_{2}$ is as Eq. (2) (Ding et al., 2012). 
$\left\{\begin{array}{l}x_{\mathrm{M}}^{(2)}=[(m-a) \cos \theta-(n \pi+n t-b) \sin \theta] \cos \frac{t+\pi}{i_{12}} \\ y_{\mathrm{M}}^{(2)}=-[(m-a) \cos \theta-(n \pi+n t-b) \sin \theta] \sin \frac{t+\pi}{i_{12}} \\ z_{\mathrm{M}}^{(2)}=-(m-a) \sin \theta-(n \pi+n t-b) \cos \theta\end{array}\right.$

where $i_{12}$ is the transmission ratio, $i_{12}=\frac{N_{2}}{N_{1}}$. As shown in Fig. 1, $a$ and $b$ are the components of the center distance from the axis $x_{1}$ and axis $z_{1}$ respectively. The position parameters for the concave convex arc line gear mechanism can be derived from Eq. (3) or (4) (Ding et al., 2014).

$$
\begin{aligned}
& \left\{\begin{array}{l}
a=\left(1+i_{12} \cos \theta\right) m \\
b=(m-a) \tan \theta
\end{array}\right. \\
& \left\{\begin{array}{l}
a=m \\
b=i_{12} m
\end{array}\right.
\end{aligned}
$$

where $\theta$ is the included angle between the angular velocity vectors of the driving and driven line gear, $\theta \in[0, \pi]$. When $\theta \neq \frac{\pi}{2}$, the values of $a$ and $b$ are derived from Eq. (3). When $\theta=\frac{\pi}{2}$, the values of $a$ and $b$ can be obtained by Eq. (4). Therefore, $i_{12}, \theta, a, b, m$ and $n$ are the key factors of the contact curve of the driven line gear.

\subsection{Parametric design formulae for the driving line gear}

A driving line gear is formed by driving line gear teeth radial attachment to a cylindrical wheel body. The gear teeth can be convex or concave, and the gear with convex teeth is taken as an example here. As shown in Fig. 2, a convex tooth of a driving line gear is formed by the motion of a convex normal profile along a driving contact curve and two tooth thickness auxiliary curves.

The two tooth thickness auxiliary curves have two functions. Firstly, they lead the movement of the auxiliary line of the gear tooth profile. Secondly, they provide the normal tooth thickness of the gear teeth. The auxiliary curves of tooth thickness comprise a first auxiliary curve and a second auxiliary curve. A first auxiliary curve 2 is arranged between a contact curve 1 and a second auxiliary curve 3 , and its equation in $o_{1}-x_{1} y_{1} z_{1}$ is as Eq. (5).

$$
\left\{\begin{array}{l}
x_{M_{11}}^{(1)}=m \cos t-\frac{c_{1} n \sin t}{\sqrt{n^{2}+m^{2}}} \\
y_{M_{11}}^{(1)}=m \sin t+\frac{c_{1} n \cos t}{\sqrt{n^{2}+m^{2}}} \\
z_{M_{11}}^{(1)}=n \pi+n t-\frac{c_{1} m}{\sqrt{n^{2}+m^{2}}}
\end{array}\right.
$$

The equation of a second auxiliary curve 3 in $o_{1}-x_{1} y_{1} z_{1}$ is as Eq. (6).

$$
\left\{\begin{array}{l}
x_{M_{12}}^{(1)}=m \cos t-\frac{2 c_{1} n \sin t}{\sqrt{n^{2}+m^{2}}} \\
y_{M_{12}}^{(1)}=m \sin t+\frac{2 c_{1} n \cos t}{\sqrt{n^{2}+m^{2}}} \\
z_{M_{12}}^{(1)}=n \pi+n t-\frac{2 c_{1} m}{\sqrt{n^{2}+m^{2}}}
\end{array}\right.
$$

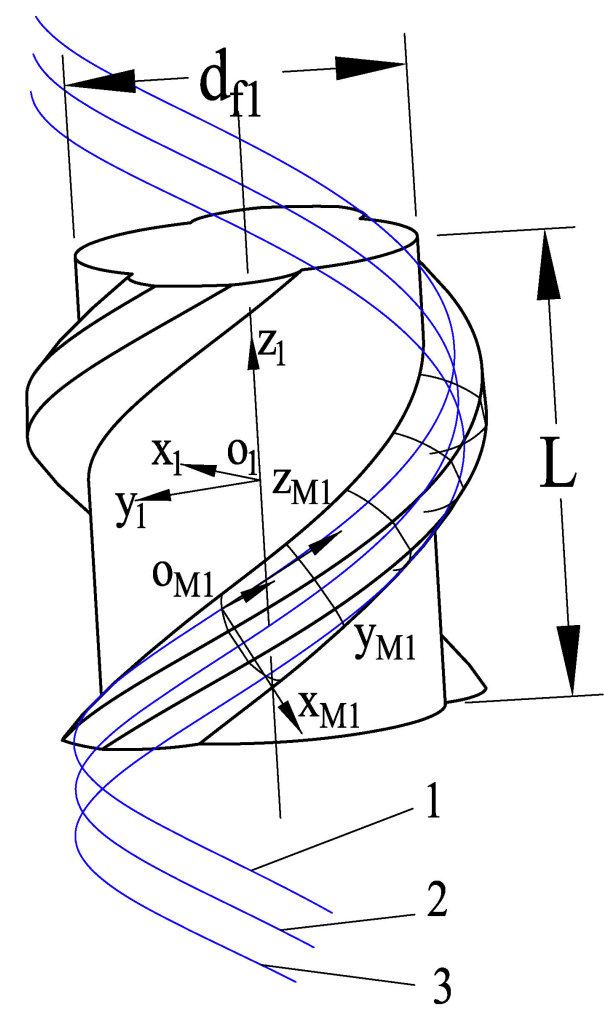

Figure 2. Schematic diagram of the driving line gear.

where, $2 c_{1}$ is the normal tooth thickness of the driving line gear.

The driving line gear is designed by determining the following parameters. Parameters of a driving line gear include driving contact curve parameters, tooth profile parameters and wheel body parameters. Driving contact curve parameters cover $m$ and $n$. Tooth profile parameters of the driving line gear are shown in Fig. 3. The tooth profile is composed of two sections of arcs and a section of straight line. The two sections of arcs are symmetrical to a first auxiliary curve of tooth thickness in the plane of the normal tooth profile, and the radius of the arc is $\rho_{1}$. At the mesh point, the angle between a straight line and $-\gamma$ is $\phi$. And the straight line is a connection between the mesh point and arc center. And $\gamma$ is the secondary normal vector of contact curve of the driving line gear. The straight line of the driving line gear tooth profile is parallel to $-\boldsymbol{\gamma}$. The distance between them is $h_{a 1}$. $h_{a 1}$ and $h_{f 1}$ are the driving line gear addendum and dedendum respectively. As shown in Fig. 2, wheel body parameters include diameter $d_{f 1}$ and length $L$. The complete driving line gear model can be established by the cylindrical wheel body and the line gear convex teeth, in which the height of the tooth is limited by the driving contact curve and $h_{a 1}$. And $h_{f 1}$ is not required to be calculated. In the engagement of a couple conjugated space curves, the contact ratio affects the curve length, indirectly determines the length of a driving 


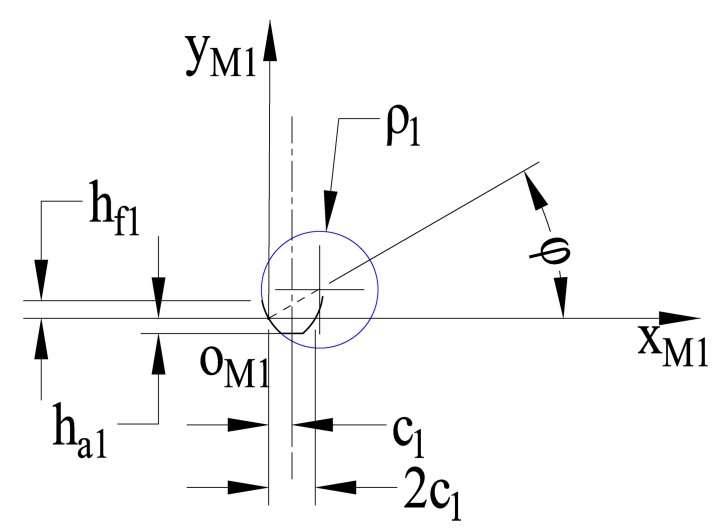

Figure 3. Schematic diagram of the normal tooth profile of the driving line gear.

line gear. Therefore, the value of $L$ is related to the contact ratio. And it depends on $t_{\mathrm{S}}$ and $t_{\mathrm{e}}$.

\subsection{Parametric design formulae of the driven line gear}

A driven line gear is formed by the driven line gear teeth radial attachment to a conical or cylindrical wheel body. The driven line gear teeth can be concave or convex, and the gear with concave teeth is taken as an example here. As shown in Fig. 4, a concave tooth of a driven line gear is formed by the motion of a concave normal profile along a driven contact curve and two tooth thickness auxiliary curves.

For the driven line gear, two tooth thickness auxiliary curves have also two functions. The auxiliary curves of driven line gear tooth thickness comprise a first auxiliary curve and a second auxiliary curve. A first auxiliary curve 2 is arranged between a driven contact curve 1 and a second auxiliary curve 3, and its equation in $o_{2}-x_{2} y_{2} z_{2}$ is as Eq. (7).

$$
\left\{\begin{array}{l}
x_{M_{21}}^{(2)}=\left[(m-a) \cos \theta-\left(n \pi+n t-b-\frac{c_{2} m}{\sqrt{n^{2}+m^{2}}}\right) \sin \theta\right] \cos \frac{t+\pi}{i_{12}}-\frac{c_{2} n}{\sqrt{n^{2}+m^{2}}} \sin \frac{t+\pi}{i_{12}} \\
y_{M_{21}}^{(2)}=-\left[(m-a) \cos \theta-\left(n \pi+n t-b-\frac{c_{2} m}{\sqrt{n^{2}+m^{2}}}\right) \sin \theta\right] \sin \frac{t+\pi}{i_{12}}-\frac{c_{2} n}{\sqrt{n^{2}+m^{2}}} \cos \frac{t+\pi}{i_{12}} \\
z_{M_{21}}^{(2)}=-(m-a) \sin \theta-\left(n \pi+n t-b-\frac{c_{2} m}{\sqrt{n^{2}+m^{2}}}\right) \cos \theta
\end{array}\right.
$$

The equation of a second auxiliary curve 3 of a driven line gear in $o_{2}-x_{2} y_{2} z_{2}$ is as Eq. (8).

$$
\left\{\begin{array}{l}
x_{M_{M_{2}}^{(2)}}=\left[(m-a) \cos \theta-\left(n \pi+n t-b-\frac{2 c_{2} m}{\sqrt{n^{2}+m^{2}}}\right) \sin \theta\right] \cos \frac{t+\pi}{i_{12}}-\frac{2 c_{2} n}{\sqrt{n^{2}+m^{2}}} \sin \frac{t+\pi}{i_{12}} \\
y_{M_{22}}^{(2)}=-\left[(m-a) \cos \theta-\left(n \pi+n t-b-\frac{2 c_{2} m}{\sqrt{n^{2}+m^{2}}}\right) \sin \theta\right] \sin \frac{t+\pi}{i_{12}}-\frac{2 c_{2} n}{\sqrt{n^{2}+m^{2}}} \cos \frac{t+\pi}{i_{12}} \\
z_{M_{22}}^{(2)}=-(m-a) \sin \theta-\left(n \pi+n t-b-\frac{2 c_{2} m}{\sqrt{n^{2}+m^{2}}}\right) \cos \theta
\end{array}\right.
$$

where, $2 c_{2}$ is the normal tooth thickness of the driven line gear.

Parameters of a driven line gear also include driven contact curve parameters, tooth profile parameters and wheel body parameters. The driven contact curve parameters cover $m, n$, $\theta, a$ and $b$. Tooth profile parameters of a driven line gear

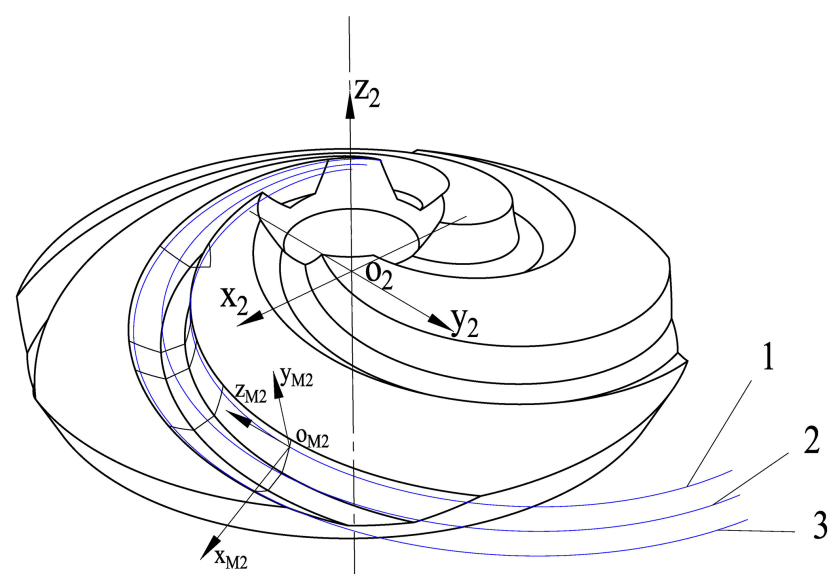

Figure 4. Schematic diagram of the driven line gear.

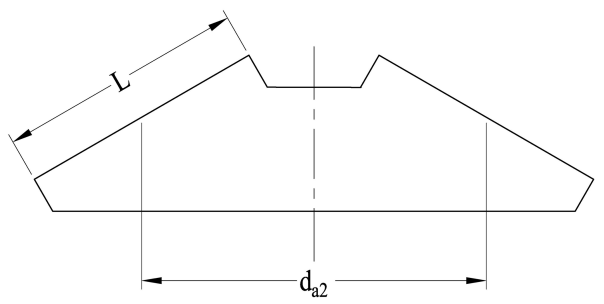

Figure 5. Rotary surface of the driven line gear.

are shown in Fig. 6. The tooth profile is composed of two sections of arcs and a section of straight line. The two sections of arcs are symmetrical to the first auxiliary curve of tooth thickness in the plane of the normal tooth profile, and the radius of the arc is $\rho_{2}$. At the mesh point, the angle between a straight line and $-\gamma$ is $\phi$. And the straight line is a connection between the mesh point and arc center. And $\gamma$ is the secondary normal vector of contact curve of the driving line gear. The straight line of the driven line gear tooth profile is parallel to $-\boldsymbol{\gamma}$, and the distance between them is $h_{f 2}$. $d_{a 2}$ and $h_{f 2}$ are the driven line gear addendum and dedendum respectively. Wheel body parameters include diameter $d_{a 2}$ and length $L$, as shown in Fig. 5. $d_{a 2}$ is the outside diameter of the driven line gear at the normal tooth profile of the middle section of the meshing curve. The complete driven line gear model can be established by the wheel body and the line gear concave teeth, in which the depth of the tooth is limited by the driven contact curve and $h_{f 2}$. And $h_{a 2}$ is not required to be calculated. But when $\theta=\frac{\pi}{2}$, the value of $d_{a 2}$ is not representative of the size of the wheel body. At this time, it is necessary to determine the value of $h_{a 2}$. In the transmission of a couple of conjugated curves, the length of a driven line gear tooth depends on the length of a driving line gear tooth. Therefore, the length of the driven line gear tooth is equal to the length of the driving line gear tooth. 


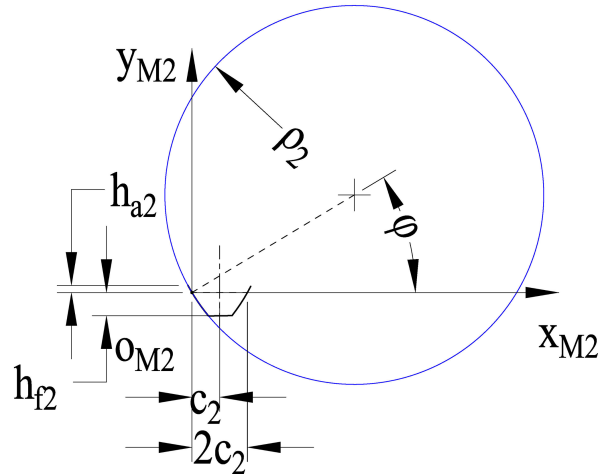

Figure 6. Schematic diagram of the normal tooth profile of the driven line gear.

\section{The optimal formulae of the tooth profile parameters of a concave convex arc line gear mechanism}

\subsection{Interference proof condition}

As shown in Fig. 7a, if only consider that the tooth profile arcs are tangent at meshing point, the actual surfaces of the driving line gear tooth and driven line gear tooth may interfere with each other outside of meshing point, resulting in abnormal engagement. The tooth surface can be considered as a set of tooth profiles on normal plane of each point at the contact curve of the driving line gear. The relationship between the driving line gear tooth profile and the driven line gear tooth profile on the normal plane of meshing point is shown in Fig. 7b-d. Coordinate system $o_{\mathrm{E}}-x_{\mathrm{E}} y_{\mathrm{E}} z_{\mathrm{E}}$ is established, which is obtained by the coordinate system $o_{\mathrm{M} 1}-x_{\mathrm{M} 1} y_{\mathrm{M} 1} z_{\mathrm{M} 1}$ moving a distance parallel to $\rho_{1}$ on the plane $x_{\mathrm{M} 1} o_{\mathrm{M} 1} y_{\mathrm{M} 1}$. The origin of system $o_{\mathrm{E}}-x_{\mathrm{E}} y_{\mathrm{E}} z_{\mathrm{E}}$ is located at the center of arc of the driving line gear tooth profile, as shown in Fig. 7b and c. Therefore, the Interference proof condition of teeth surfaces is that the distance between $o_{\mathrm{E}}$ and any point of driven line gear tooth profile arc is not less than $\rho_{1}$.

\subsection{Transformation of the coordinate systems}

The origin of the coordinate system $o_{\mathrm{M} 1}-x_{\mathrm{M} 1} y_{\mathrm{M} 1} z_{\mathrm{M} 1}$ is located on the contact curve of the driving line gear, and the directions of each coordinate axis are $-\boldsymbol{\gamma}, \boldsymbol{\beta}$ and $\boldsymbol{\alpha}$. Where, $\boldsymbol{\alpha}, \boldsymbol{\beta}$ and $\boldsymbol{\gamma}$ are the tangent vector, normal vector and secondly normal vector of the contact curve of the driving line gear respectively. The equation of the driven line gear tooth surface is obtained through the coordinate transformation of $o_{\mathrm{E}}-x_{\mathrm{E}} y_{\mathrm{E}} z_{\mathrm{E}}$ and $o_{2}-x_{2} y_{2} z_{2}$, which is as Eq. (9).

$$
\left[\begin{array}{c}
X \\
Y \\
Z \\
1
\end{array}\right]=M_{e 2}\left[\begin{array}{c}
x \\
y \\
z \\
1
\end{array}\right]
$$

The equation of $M_{e 2}$ is as Eq. (10).

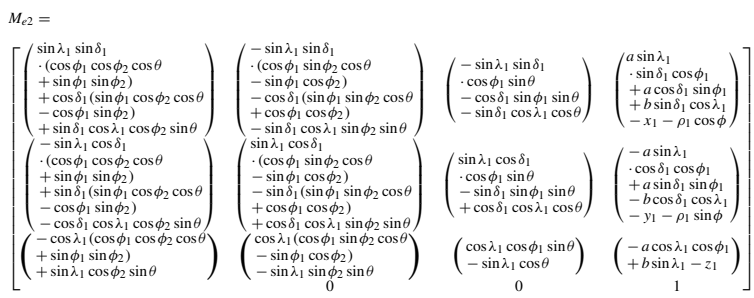

where $\phi_{1}=t+\pi ; \phi_{2}=\frac{t+\pi}{i_{12}} ; \lambda_{1}$ is the helix angle of contact curve of the driving line gear, $\lambda_{1}=\arctan \frac{n}{m} ; \delta_{1}$ is the included angle of $\boldsymbol{\beta}$ and axis $x_{1}, \delta_{1}=\arccos \left(\frac{\left(x_{1}, y_{1}, 0\right) \cdot(1,0,0)}{\left|\left(x_{1}, y_{1}, 0\right)\right||(1,0,0)|}\right)$; $x_{1}, y_{1}$ and $z_{1}$ are the values of the coordinate origin $o_{\mathrm{M} 1}$ in $o_{1}-x_{1} y_{1} z_{1}$.

\subsection{The equation of the main tooth surface of the driven line gear}

The tooth surface of the driven line gear is formed by a circular arc with a radius of $\rho_{2}$ along the three space curves. At the initial position, the center of the circle arc is at point $\left(x_{\mathrm{c}}, y_{\mathrm{c}}\right.$, $\left.z_{\mathrm{c}}\right)$, which is obtained by coordinate transformation of $\left(x_{2 s}\right.$, $\left.y_{2 s}, z_{2 s}\right)$, the equation is as Eq. (11).

$\begin{cases}x_{\mathrm{C}} & =x_{2 s}+\rho_{2} \sin \phi=\left[(m-a) \cos \theta-\left(n \pi+n t_{\mathrm{S}}-b\right) \sin \theta\right] \cos \frac{t_{\mathrm{S}}+\pi}{i_{12}} \\ y_{\mathrm{C}} & =y_{2 s}-\rho_{2} \cos \phi \sin \lambda_{2}=-\left[(m-a) \cos \theta-\left(n \pi+n t_{\mathrm{S}}-b\right) \sin \theta\right] \sin \frac{t_{\mathrm{S}}+\pi}{i_{12}} \\ z_{\mathrm{c}} & =z_{2 s}-\rho_{2} \cos \phi \cos \lambda_{2}=-(m-a) \sin \theta-\left(n \pi+n t_{\mathrm{S}}-b\right) \cos \theta\end{cases}$

where, $\lambda_{2}$ is the helix angle of the contact curve of driven line gear.

Taking $u$ as a parameter variable, the equation of the arc is as Eq. (12).

$\left\{\begin{array}{l}x_{0}=x_{\mathrm{c}}+\rho_{2} \cos u \\ y_{0}=y_{\mathrm{c}}-\rho_{2} \sin u \sin \lambda_{2} \\ z_{0}=z_{\mathrm{c}}-\rho_{2} \sin u \cos \lambda_{2}\end{array}\right.$

The equation of the driven line gear tooth surface in $o_{2}-x_{2} y_{2} z_{2}$ is as Eq. (13).

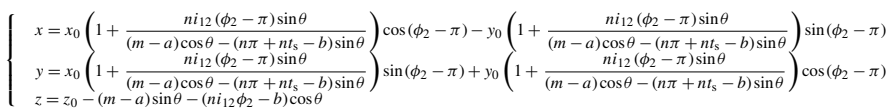

Substituting Eq. (13) into Eq. (9), the equation of driven line gear tooth surface in $o_{\mathrm{E}}-x_{\mathrm{E}} y_{\mathrm{E}} z_{\mathrm{E}}$ is obtained.

\subsection{Parameter optimization}

From Fig. 7c, d and Eq. (9), the interference proof condition of tooth surfaces between a driving line gear and a driven line gear is described as Eqs. (14) and (15). 

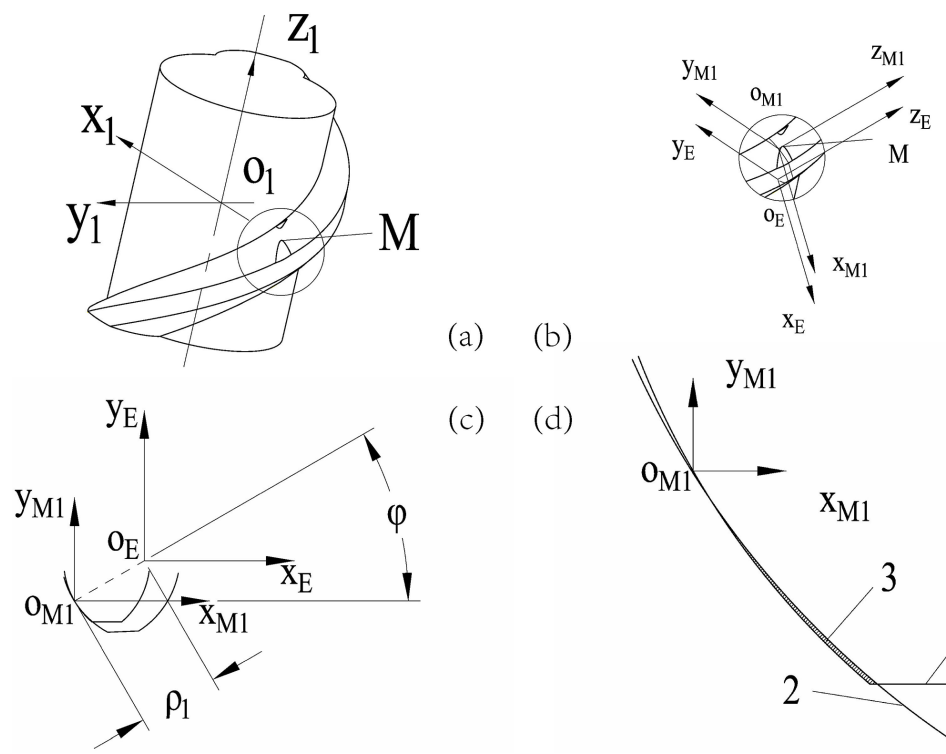

(b)

(c)

(d)

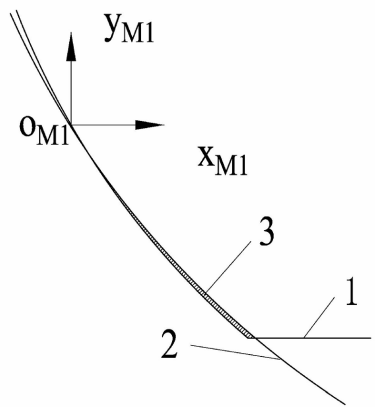

Figure 7. Diagram of the tooth profiles interference of the driving line gear and the driven line gear. $(1-$ the tooth profile arc of the driving line gear; 2 - the tooth profile arc of driven line gear on the normal plane of the contact curve of the driving line gear; 3 - an interference area.)

$$
\begin{aligned}
& \left\{\begin{array}{l}
X<-\sqrt{\rho_{1}^{2}-\left(-\rho_{1} \sin \phi-h_{a 1}\right)^{2}} \\
Y=-\rho_{1} \sin \phi-h_{a 1} \\
Z=0
\end{array}\right. \\
& \left\{\begin{array}{l}
X<-\sqrt{\rho_{1}^{2}-\left(-\rho_{1} \sin \phi+h_{a 1}\right)^{2}} \\
Y=-\rho_{1} \sin \phi+h_{a 1} \\
Z=0
\end{array}\right.
\end{aligned}
$$

Considering of interference proof, the tooth profile parameters and the wheel body parameters for the driving line gear and driven line gear can be optimized by combining Eqs. (14) and (15). And, $\rho_{2}=k \rho_{1}$. Then, the results can be drawn as follows.

If $\phi \in\left[\frac{\pi}{6}, \frac{2 \pi}{9}\right]$, Then, $\rho_{1}=\frac{1.1 c_{1}}{\cos \phi}, k \in\left(0, \frac{1}{4}\right)$, or $\rho_{1}=\frac{1.2 c_{1}}{\cos \phi}$, $k \in\left(0, \frac{1}{5}\right)$, or $\rho_{1}=\frac{1.4 c_{1}}{\cos \phi}, k \in\left(0, \frac{1}{7}\right)$, or $\rho_{1}=\frac{1.6 c_{1}}{\cos \phi}, k \in\left(0, \frac{1}{11}\right)$.

If $\phi \in\left[\frac{2 \pi}{9}, \frac{\pi}{4}\right]$, Then, $\rho_{1}=\frac{1.4 c_{1}}{\cos \phi}, k \in\left(0, \frac{1}{7}\right)$, or $\rho_{1}=\frac{1.6 c_{1}}{\cos \phi}$, $k \in\left(0, \frac{1}{11}\right)$.

In the design of line gears, the teeth clearance should also be considered. In the meshing process of the convex and concave teeth, the height of the top of the convex tooth is a priority. As shown in Fig. 3, the addendum of the driving line gear can be estimated by as Eq. (16).

$h_{a 1}=h_{\mathrm{a}}^{*} \cdot \rho_{1}(1-\sin \phi)$

where, $h_{\mathrm{a}}^{*}$ is a coefficient of addendum, and its value is within a range of $0.8-0.97$. This range is obtained by maintaining the profile shape of the convex tooth profile, according to simulation results.
And, a design formula of the dedendum of the driven line gear is obtained as Eq. (17).

$h_{f 2}=h_{f}^{*} \cdot h_{a 1}$

where, $h_{f}^{*}$ is a coefficient of dedendum, and its value is within a range of 1.4-2. The range of the value can make the driven gear tooth root and the driving gear tooth top not interfere with each other in the meshing process, according to simulation results.

The size of the wheel is designed to satisfy the in-Eq. (18).

$$
\begin{cases}\frac{d_{f 1}}{2}+\frac{d_{a 2}}{2} \cos (\pi-\theta)<a & \theta \neq \frac{\pi}{2} \\ \frac{d_{f 1}}{2}+h_{a 2}<m & \theta=\frac{\pi}{2}\end{cases}
$$

The concave convex arc line gear pair design needs to consider three non-interfering aspects. They are the noninterference of teeth surfaces, the non-interference of the tooth top and the tooth bottom, and the non-interference of the wheel body.

\section{Design examples}

Take three pairs of line gears as design examples, the values of various parameters are shown as Table 1 . The values of $\theta$ in Table 1 are $\frac{2 \pi}{3}, \frac{\pi}{2}, \pi$, respectively. The value of $\phi$ is $\frac{\pi}{6}$.

By the parameters in Table 1, three sets of line gear mechanisms are obtained, as shown in Figs. 8-10 respectively. From Table 1 and the figures, three pairs of line gear mechanisms have the same driving line gear. And the 3-D kinematic 


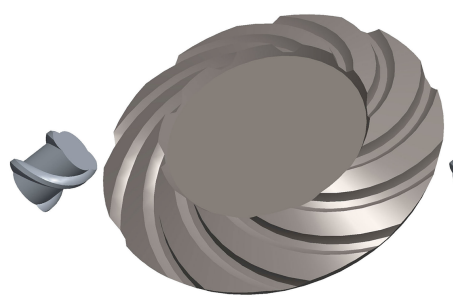

(a)

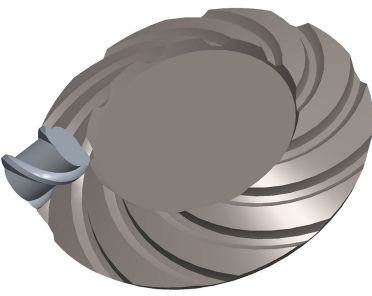

Figure 8. 3-D solid model 1. (a) Driving gear 1; (b) driven gear 1; (c) meshing simulation.

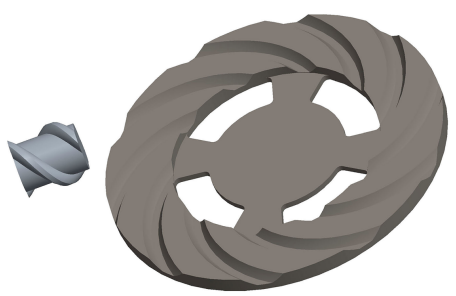

(a)

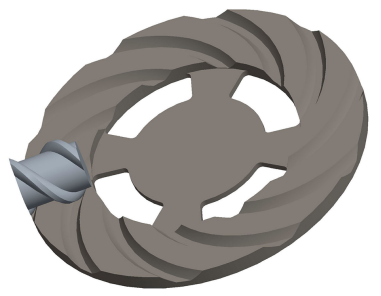

(c)

Figure 9. 3-D solid model 2. (a) Driving gear 2; (b) driven gear 2; (c) meshing simulation.

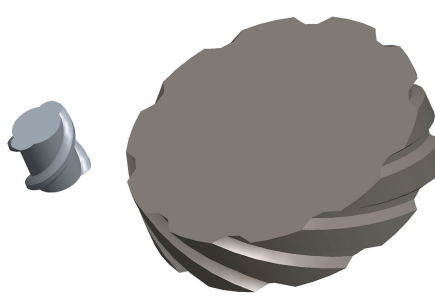

(a)

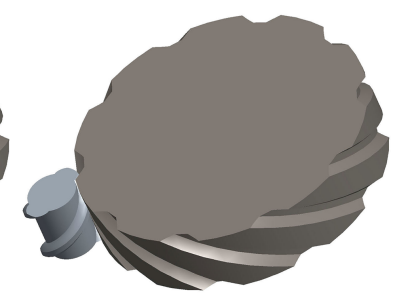

(c)
Figure 10. 3-D solid model 3. (a) Driving gear 3; (b) driven gear 3; (c) meshing simulation.

simulations on them were carried out smoothly, without interference. On one hand, it verifies the feasibility of the design formulae of parameters. On the other hand, compared with the traditional gear pair, the novel line gear mechanism has a better replacement.

Compared with other circular arc gears, the concave convex arc line gears can drive with two arbitrary intersecting axes. In the design of the concave convex arc line gear pair, the same driving wheel can be engaged with different driven wheels to drive with a variable crossed axes angle. Due to concave convex arc tooth profile, the concave convex arc line
Table 1. Parameters of three line gear pairs.

\begin{tabular}{lrrrl}
\hline Parameters & $\begin{array}{r}\text { Line } \\
\text { gear 1 }\end{array}$ & $\begin{array}{r}\text { Line } \\
\text { gear 2 }\end{array}$ & $\begin{array}{r}\text { Line } \\
\text { gear 3 }\end{array}$ & Units \\
\hline$m$ & 20 & 20 & 20 & $\mathrm{~mm}$ \\
$n$ & 12 & 12 & 12 & $\mathrm{~mm}$ \\
$i_{12}$ & 5 & 5 & 5 & - \\
$\theta$ & $\frac{2 \pi}{3}$ & $\frac{\pi}{2}$ & $\pi$ & $\mathrm{rad}$ \\
$a$ & 70 & 20 & 120 & $\mathrm{~mm}$ \\
$b$ & 86.6 & 100 & 0 & $\mathrm{~mm}$ \\
$\varepsilon$ & 1.25 & 1.25 & 1.25 & - \\
$t_{\mathrm{S}}$ & $-\frac{13 \pi}{8}$ & $-\frac{13 \pi}{8}$ & $-\frac{13 \pi}{8}$ & - \\
$t_{\mathrm{e}}$ & $-\frac{3 \pi}{8}$ & $-\frac{3 \pi}{8}$ & $-\frac{3 \pi}{8}$ & - \\
$\phi$ & $\frac{\pi}{6}$ & $\frac{\pi}{6}$ & $\frac{\pi}{6}$ & $\mathrm{rad}$ \\
$c_{1}$ & 4 & 4 & 4 & $\mathrm{~mm}$ \\
$c_{2}$ & 7 & 7 & 7 & $\mathrm{~mm}$ \\
$\rho_{1}$ & 10 & 10 & 10 & $\mathrm{~mm}$ \\
$\rho_{2}$ & 40 & 40 & 40 & $\mathrm{~mm}$ \\
$h_{a 1}$ & 2.59 & 2.59 & 2.59 & $\mathrm{~mm}$ \\
$h_{f 2}$ & 5 & 5 & 5 & $\mathrm{~mm}$ \\
\hline
\end{tabular}

gear pair is better than the early form of line gear in the bearing contact stress. Due to point contact engagement, it bears lower contact stress than the other conventional forms of arc gear pair with line contact engagement. The tooth number of concave convex arc line gear can reach 1 . The concave convex arc line gear pair has large transmission ratio and compact structure.

\section{Conclusions}

In this paper, a design method for a concave convex curve line gear mechanism is presented. And the design formulae of parameters of the tooth profile are deduced on the basis of interference proof condition proposed. Conclusions can be drawn as follows.

It is easy to mass production. The teeth of the driving line gear and driven line gear are attached to the wheel body radially. And this kind of concave convex curve line gear is easy to be processed by the numerical control machine tool, so that it is easy to mass production.

It has good replacement. For the transmission of a line gear pair with an arbitrary angle in the same plane, only one of driving line gear needs to be designed, which can match to many of driven line gear with different geometric parameters or arbitrary crossed angles. Therefore, compared with the traditional gear pairs and the other arc gear pairs, this kind of concave convex curve line gear mechanism has a good replacement.

It has power transmission capacity. Compared with previous proposed line gear mechanism, this kind of concave convex curve line gear can bear much greater power transmission capacity according to Hertz contact theory, but smaller 
than that of traditional concave convex surface gear pair due to its point contact meshing principle.

It has compact structure. The least number of driving line gear teeth of this kind of concave convex curve line gear mechanism can reach 1 . Compared with the traditional gear pair, such as spur gear pair or helical gear pair, its structure is much more compact, that can greatly save installation space.

Compared with the traditional gear pairs and the other arc gears, the characteristic of concave convex arc line gears is the space curve meshing theory. It makes the line gear have good replacement and compact structure. Compared with the early form of line gears, the characteristics of the concave convex arc line gears are the tooth profile and the relationship between the wheel body and line gear tooth. On the one hand, it is strengthened in the tooth contact strength and the bending strength of the gear tooth. This makes it available for use in the field of conventional power transmission. And that allows the transmission with a larger power. On the other hand, it can be processed by the metal cutting machine tool. And that is more convenient for efficient processing of concave convex arc line gear and the expansion of its application. The tooth profile design and tooth formation are considered by the tool shape and the motion trajectory of the machine tool. The appearance of special NC machine tool for the concave convex arc line gear can greatly improve the machining efficiency.
The remainder of this work is to design and make a special-purpose numerical control machine tool for the line gear, to facilitate the efficient processing of gear, to expand the scope of application. We are doing these research works on contact pressure, sliding speeds and efficiency, conducted by newly test rig on transmission ability and dynamic performance experiments. 


\section{Appendix A: Notation and units}

$a, b \quad$ Distances from $o_{1}$ to axis $x_{1}$ and axis $z_{1}$, [mm]

$c_{1}, c_{2} \quad$ Normal tooth thickness of the driving and driven line gear, [mm]

$d_{a 2} \quad$ Outside diameter of the driven line gear at the normal tooth profile of the middle section of

the meshing curve, [mm]

$d_{f 1} \quad$ Diameter of the driving line gear wheel body, [mm]

$h_{\mathrm{a}}^{*} \quad$ Coefficient of addendum, [-]

$h_{a 1}, h_{a 2} \quad$ Addendums of the driving and driven line gear, [mm]

$h_{f}^{*} \quad$ Coefficient of dedendum, [-]

$h_{f 1}, h_{f 2}$ Dedendums of the driving and driven line gear, [mm]

$i_{12} \quad$ Ttransmission ratio, [-]

$k \quad$ Coefficient associated with $\rho_{2},[-]$

$L \quad$ Length of the driving line gear wheel body, [mm]

$m \quad$ Helix radius of the cylindrical spiral curve, [mm]

$n \quad$ Pitch parameter of the cylindrical spiral curve, $[\mathrm{mm}]$

$N_{1}, N_{2} \quad$ Tooth numbers of the driving and driven line gear, [-]

$p \quad$ Pitch of the cylindrical spiral curve, $[\mathrm{mm}]$

$t \quad$ Parameter variable, $[-]$

$t_{\mathrm{S}}, t_{\mathrm{e}} \quad$ Values of $t$ at initial and terminal meshing points, [-]

$\Delta t \quad$ Difference from initial meshing point to terminal meshing point, [-]

$u \quad$ Parameter variable, $[-]$

$\boldsymbol{\alpha} \quad$ Tangent vector of the driving contact curve, $[-]$

$\boldsymbol{\beta} \quad$ Normal vector of the driving contact curve, $[-]$

$\gamma \quad$ Secondly normal vector of the driving contact curve, $[-]$

$\delta_{1} \quad$ Included angle of $\boldsymbol{\beta}$ and axis $x_{1}$, [rad]

$\varepsilon \quad$ Contact ratio, $[-]$

$\theta \quad$ Included angle between the angular velocity vectors of the driving and driven line gear, [rad]

$\lambda_{1}, \lambda_{2} \quad$ Helix angles of the driving and driven contact curve, [rad]

$\rho_{1}, \rho_{2} \quad$ Radius of the driving and driven tooth profile arc, [mm]

$\phi \quad$ Angle between $-\boldsymbol{\gamma}$ and a line passing through tooth profile arc center and $o_{\mathrm{M}}$, [rad]

$\phi_{1}, \phi_{2} \quad$ Included angles of the driving and driven contact curves, [rad] 
Acknowledgements. Funding supports from the National Natural Science Foundation of China (No. 51175180, No. 51575191) are gratefully acknowledged.

Edited by: D. Pisla

Reviewed by: three anonymous referees

\section{References}

Chen, B. K., Liang, D., and Gao, Y.: Geometry design and mathematical model of a new kind of gear transmission with circular arc tooth profiles based on curve contact analysis, Proc. Inst. Mech. Eng. Part C-J. Eng. Mech. Eng. Sci., 228, 3200-3208, doi:10.1177/0954406214526866, 2014.

Chen, H. J., Zhang, X. P., Cai, X., Ju, Z. L., Qu, C., and Shi, D. H.: Computerized design, generation and simulation of meshing and contact of hyperboloidal-type normal circular-arc gears, Mech. Mach. Theory, 96, 127-145, doi:10.1016/j.mechmachtheory.2015.08.022, 2016.

Chen, Y. Z.: Line Gear, Science Press of China, Beijing, China, 2014.

Chen, Y. Z. and Yao, L.: A kind of gear transmission with parallel axes and concave convex arc tooth, China, ZL201510570926.9, PCT/CN2015/100182, http://d.g.wanfangdata.com.cn/Patent_CN201510570926.9.aspx (last access: 31 December 2015), 2015.

Chen, Y. Z., Chen, Z., Zhang, Y., and Asme: Contact ratio of spatial helix gearing mechanism, International Mechanical Engineering Congress And Exposition - 2012, Vol. 3, Pts a-c: Design, Materials, And Manufacturing, Amer. Soc. Mechanical Engineers, New York, 1529-1536, 2013a.

Chen, Y. Z., Lv, Y. L., Ding, J., and Chen, Z.: Fundamental design equations for space curve meshing skew gear mechanism, Mech. Mach. Theory, 70, 175-188, doi:10.1016/j.mechmachtheory.2013.07.004, 2013 b.

Ding, J., Chen, Y. Z., and Lv, Y. L.: Design of Space-Curve Meshing-Wheels with Unequal Tine Radii, Strojniski Vestn.-J. Mech. Eng., 58, 633-641, doi:10.5545/sv-jme.2012.493, 2012.

Ding, J., Chen, Y. Z., Lv, Y. L., and Song, C. H.: Position-Parameter Selection Criterion for a Helix-Curve Meshing-Wheel Mechanism Based on Sliding Rates, Strojniski Vestn.-J. Mech. Eng., 60, 561-570, doi:10.5545/sv-jme.2013.1574, 2014.

Dong, G. J. and Wang, M.: Modeling of Error Analysis Simulation of Normal Circular Arc Bevel Gear Transmission, in: Advances In Machining And Manufacturing Technology Xii, edited by: Xu, X., Huang, C., Zuo, D., and Chen, M., Key Engineering Materials, Trans Tech Publications Ltd, Stafa, Zurich, 606-610, 2014.
Ren, Z. Y.: MeshingAnalysis of Quadruple-arc Profile Bevel Gears in the Process of Cutting Tooth, in: Engineering Solutions for Manufacturing Processes Iv, Pts. 1 and 2, Advanced Materials Research, edited by: Jiang, Z., Liu, X., and Han, J., Trans. Tech. Publications Ltd, Stafa, Zurich, 507-512, 2014.

Sun, Z. J., Hou, L., Wang, J. E., Li, W., and Chang, Q. L.: Contact strength analysis of circular-arc-tooth-trace cylindrical gear, J. Braz. Soc. Mech. Sci. Eng., 38, 999-1005, doi:10.1007/s40430014-0272-6, 2016.

Wang, C. Y.: Design of Planetary Gear Reducer with Double Circular-Arc Helical Gear, in: Mechanical Engineering And Materials, Pts. 1-3, Applied Mechanics and Materials, Trans. Tech. Publications Ltd, Stafa, Zurich, 1595-1600, 2012.

Xie, X. S. and Yang, H. C.: Kinematic errors on helical gear of triple circular-arc teeth, J. Mech. Sci. Technol., 28, 3137-3146, doi:10.1007/s12206-014-0722-1, 2014.

Zhang, R. L., Wang, T., and Li, H. M.: Tooth Contact Analysis of the Double Circular Arc Tooth Spiral Bevel Gear, in: Frontiers Of Manufacturing And Design Science, Pts. 1-4, Applied Mechanics and Materials, edited by: Chen, R., Trans. Tech. Publications Ltd, Stafa, Zurich, 3711-3715, 2011.

Zhang, R. L., Wang, T., and Wu, Z. F.: TCA Principe and Application of the Double Circular Arc Tooth Spiral Bevel Gear, in: Frontiers Of Manufacturing And Design Science II, Pts. 1-6, Applied Mechanics and Materials, edited by: Sun, D., Sung, W. P., and Chen, R., Trans. Tech. Publications Ltd, Stafa, Zurich, 35593561, 2012.

Zhang, X. G., Xie, Y. C., and Tan, X. Q.: Design, meshing characteristics and stress analysis of cylindrical gears with curvilinear tooth profile, Trans. Famena, 40, 27-44, 2016.

Zhao, Y. P. and Zhang, Z.: Mathematical Model of Manufacturing a Novel Type of Hourglass Worm with Circular Tooth Profiles, in: Advanced Design And Manufacture III, edited by: Su, D. Z., Zhang, Q. B., and Zhu, S. F., Trans. Tech. Publications Ltd, Stafa, Zurich, 337-340 pp., 2011.

Zhou, Y., Hao, S. H., and Hao, M. H.: Design and performance analysis of a circular-arc gear pump operating at high pressure and high speed, Proc. Inst. Mech. Eng. Pt. C, 230, 189-205, doi:10.1177/0954406215572435, 2016. 